\title{
PERICARDIAL SCLEROSIS AS THE PRIMARY MANAGEMENT OF MALIGNANT PERICARDIAL EFFUSION AND CARDIAC TAMPONADE
}

Elizabeth A. Maher, MD, $\mathrm{PhD}^{\mathrm{a}}$

Frances A. Shepherd, MD, FRCPC

Thomas J. R. Todd, MD, FRCSC ${ }^{\text {b }}$
Objective: The management of malignant pericardial effusion remains controversial. We present our experience with 93 patients referred for drainage and sclerosing procedures between 1979 and 1994. Methods: With continuous electrocardiographic monitoring, a Kifa catheter was inserted percutaneously into the pericardial sac and allowed to drain. A $100 \mathrm{mg}$ dose of lidocaine hydrochloride was instilled intrapericardially, followed by $\mathbf{5 0 0}$ to $1000 \mathrm{mg}$ tetracycline or doxycycline hydrochloride in 20 to $50 \mathrm{ml}$ normal saline solution. The catheter was clamped for 1 to 2 hours and then reopened, and the procedure was repeated daily until the net drainage was less than $25 \mathrm{ml}$ in 24 hours. Results: Subjects included 53 women and 40 men (median age 58 years). Eight patients could not undergo sclerosis because of technical failure. Eighty-five patients underwent sclerosis and required a median dose of $1500 \mathrm{mg}$ of the sclerosing agent (range 500 to 700 $\mathrm{mg}$ ), given in a median of three injections (range one to eight). Complications included pain (17 patients), atrial arrhythmias (eight patients), fever with temperature greater than $38.5^{\circ} \mathrm{C}$ (seven patients), and infection (one patient). Two patients had cardiac arrest before sclerosis could be attempted. Sixty-eight patients (73\%) had the effusion controlled for longer than 30 days, for an overall control rate of $81 \%$. Seven other patients had control of the effusion but died of progressive malignant disease in less than 30 days. The overall median survival was 98 days (range 1 to 1724 days). Comparison of these results with outcomes reported for patients with malignant pericardial effusion who underwent surgical drainage indicates that drainage and sclerosis provide similar survivals but sclerosis carries lower morbidity, mortality, and recurrence rates. Conclusion: Percutaneous drainage and sclerosis constitutes a safe and effective treatment for malignant pericardial effusion. Surgical management should be reserved for the small percentage of cases that cannot be controlled by this method. (J Thorac Cardiovasc Surg 1996;112:637-43)
$\mathrm{T}$ he incidence of pericardial involvement as a complication of malignancy has been reported to range from as low as $0.1 \%$ in clinical studies to $21 \%$ in some autopsy series. ${ }^{1}$ Malignant pericardial effu-

From the Department of Medicine, Division of Hematology/ Oncology, ${ }^{\mathrm{a}}$ and the Department of Surgery, Division of Thoracic Surgery, ${ }^{\mathrm{b}}$ The Toronto Hospital and the University of Toronto, Toronto, Ontario, Canada.

Received for publication Jan. 2, 1996; revisions requested Feb. 16, 1996; revisions received March 13, 1996; accepted for publication March 18, 1996.

Address for reprints: Frances A. Shepherd, MD, FRCPC, The Toronto Hospital, ML2-035, 200 Elizabeth St., Toronto, Ontario, Canada M5G $2 \mathrm{C} 4$.

Copyright (C) 1996 by Mosby-Year Book, Inc.

$0022-5223 / 96 \$ 5.00+0 \quad \mathbf{1 2} / \mathbf{1} / \mathbf{7 3 5 3 0}$ sion (MPE) is frequently not suspected until the signs and symptoms of cardiac tamponade develop; at this stage, this complication of malignancy is potentially fatal unless promptly treated. Treatment of this oncologic emergency, however, often results in good palliation of symptoms and prolongation of survival. $^{2}$

Several studies have shown that tetracycline sclerosis is a safe and effective treatment for MPE., Recent reports, however, continue to suggest that a surgical approach, either subxiphoid drainage with or without window formation or a limited pericardiotomy, should be the initial treatment for MPE. ${ }^{5-7}$ The development of thoracoscopy has permitted more ready surgical access to the pericardium, and recent reports recommend this approach. ${ }^{8,9}$ For the 
Table I. Characteristics of 93 patients with MPE

\begin{tabular}{lcc}
\hline & No. & $\%$ \\
\hline Sex & & \\
Male & 40 & 43 \\
Female & 53 & 57 \\
Age (yr) & & \\
Median & 58 & \\
Range & $24-77$ & \\
Primary tumor & & \\
Lung & 45 & 48 \\
Breast & 26 & 28 \\
Adenocarcinoma of unknown origin & 8 & 9 \\
Mesothelioma & 6 & 7 \\
Stomach & 3 & 3 \\
Lymphoma & 2 & 2 \\
Leukemia & 1 & 1 \\
Ovary & 1 & 1 \\
Sarcoma & 1 & 1 \\
Cytologic examination & & \\
Positive & 69 & 77 \\
Negative & 21 & 23 \\
Not done & 3 & 3 \\
\hline
\end{tabular}

past 15 years, patients with cardiac tamponade arising from MPE have been treated medically in our institution with percutaneous drainage and tetracycline sclerosis of the pericardial sac. We present here our updated experience with tetracycline sclerosis and compare our results with outcomes reported in the surgical literature.

\section{Methods}

We reviewed the records of 99 patients with MPE seen at our institution between January 1, 1979, and March 1, 1994. Of these patients, 93 were referred for drainage and tetracycline or doxycycline sclerosis of the pericardial space.

Baseline investigations for all patients included history, physical examination, chest radiography, electrocardiography, and two-dimensional echocardiography. After echocardiographic confirmation of the presence of pericardial effusion, patients were taken to the cardiac care unit for electrocardiographic monitoring. Under sterile conditions, a $6.5 \mathrm{~F}$ Kifa catheter (Cook Incorporated, Bloomington, Ind.) was inserted percutaneously through a subxiphoid approach into the pericardial sac by means of the Seldinger technique. The catheter was then connected to a Hemovac system (Zimmer, Patient Care Div., Dover, Ohio) with a three-way stopcock and left in place to drain.

When patients were in hemodynamically stable condition, they were transferred to the general medical ward for the sclerosing procedure. To prevent pain, $100 \mathrm{mg}$ lidocaine hydrochloride was injected intrapericardially before instillation of the sclerosing agent. Either 500 to $1000 \mathrm{mg}$ tetracycline hydrochloride (80 patients) or 500 to $1000 \mathrm{mg}$ doxycycline hydrochloride (five patients) was dissolved in 10 to $20 \mathrm{ml}$ normal saline solution and inserted through the catheter into the pericardial sac. The catheter was clamped for 1 to 2 hours and then reopened and allowed to drain to the Hemovac system. Special positioning of the patient was not required. This procedure was repeated every 24 to 48 hours, until the net drainage was less than $25 \mathrm{ml} / 24$ hours. The catheter was removed only after echocardiography confirmed that the effusion had resolved. Patient follow-up after sclerosis was predominantly by means of physical examination and chest radiography. Echocardiography was repeated as clinically indicated.

Pericardial fluid from the initial pericardiocentesis was examined for protein, cell count, cytologic characteristics, and culture. Treatment outcome was evaluated according to the criteria of Smith, Lane, and Hudgins. ${ }^{10}$ Successful treatment was defined as follows: (1) reduction or resolution of the effusion for a minimum of 30 days, (2) the absence of symptoms, and (3) no requirement for pericardiocentesis within 30 days of initiation of treatment. Survival time was calculated from the date of first instillation of the sclerosing agent to the date of death or most recent follow-up.

\section{Results}

Of the 99 patients, three underwent pericardiocentesis alone and three who had neither clinical nor echocardiographic evidence of tamponade did not have their effusions drained. The remaining 93 patients were referred for tetracycline or doxycycline sclerosis and form the basis of this report.

The characteristics of the study population are presented in Table I. There were 53 women and 40 men, with a median age of 58 years (range 24 to 77 years). Carcinoma of the breast and lung accounted for $76 \%$ of the primary tumors. The remaining $24 \%$ included adenocarcinoma of unknown origin in eight patients $(9 \%)$, mesothelioma in six patients $(7 \%)$, stomach cancer in three patients $(3 \%)$, and non-Hodgkins lymphoma, Hodgkins lymphoma, leukemia, ovarian cancer, and sarcoma in one patient each. MPE was diagnosed at the initial presentation of the malignancy in two patients with lung cancer, but MPE was diagnosed several months to several years after the first cancer diagnosis in the remaining patients. The pericardial fluid was examined cytologically in 90 patients and had malignant cells in $77 \%$ of these cases. All baseline pericardial fluid samples were sterile.

Eight patients had received previous treatment for their pericardial effusions. Five patients had reaccumulation of fluid after therapeutic pericardiocentesis without sclerosis, and three patients were treated after failed surgical drainage procedures. One of the surgical patients had undergone both simple pericardiocentesis and partial pericardiectomy. A second patient, who had had subxiphoid pericardial windows created on two occasions, un- 
Table II. Clinical presentation of MPE

\begin{tabular}{lrr}
\hline & No. & $\%$ \\
\hline Symptoms & & \\
Dyspnea & 85 & 91 \\
Cough & 39 & 42 \\
Orthopnea & 30 & 32 \\
Chest pain & 19 & 20 \\
Peripheral edema & 16 & 17 \\
Nausea & 11 & 12 \\
Impaired level of consciousness & 5 & 5 \\
Diaphoresis & 4 & 4 \\
Dysphagia & 3 & 3 \\
Hemoptysis & 2 & 2 \\
Syncope & 2 & 2 \\
Facial swelling & 2 & 2 \\
Signs & & \\
Paradoxic pulse (>10 mm Hg) & 58 & 62 \\
Elevated jugular venous pressure & 47 & 51 \\
Tachycardia $(>110$ beats/min) & 40 & 43 \\
Systolic BP (<110 mm Hg) & 39 & 42 \\
Respiratory rate (>20 breaths/min) & 33 & 35 \\
Kussmal's sign & 13 & 14 \\
Friction rub & 6 & 6 \\
Hepatomegaly & 2 & 2 \\
&
\end{tabular}

$B P$, Blood pressure.

derwent sclerosis when cardiac tamponade developed as a result of a recurrent loculated effusion. The third patient had persistent drainage from two surgically placed large-bore indwelling drainage tubes.

Dyspnea, the most common presenting symptom of MPE, was found in 85 patients (91\%; Table II). Other common symptoms included cough in 39 patients $(42 \%)$, orthopnea in 30 patients $(32 \%)$, chest pain in 19 patients $(20 \%)$ and peripheral edema in 16 patients (17\%). When first seen, all patients had one or more physical findings suggestive of cardiac tamponade, with paradoxic pulse (62\% of patients) and elevated jugular venous pressure ( $51 \%$ of patients) being the most common. The diagnosis of MPE was confirmed by two-dimensional echocardiography for all patients (Table III). Right atrial inversion was present in 61 patients $(66 \%)$ and right ventricular collapse was present in 46 patients (49\%); both are evidence of cardiac tamponade. Pericardial metastases were identified in 19 patients $(20 \%)$.

The classic electrocardiographic finding of electrical alternans was seen in only four cases; the most common finding on electrocardiography was low voltage in the limb leads (total amplitude of the QRS complex $<5 \mathrm{~mm}$ ), which was seen in 62 patients $(67 \%)$. The most common findings on chest
Table III. Investigation of $M P E$

\begin{tabular}{lrr}
\hline & No. & \% \\
\hline Echocardiography & & \\
Moderate to large MPE & 93 & 100 \\
Right atrial inversion & 61 & 66 \\
Right ventricular collapse & 46 & 49 \\
Pericardial metastases & 19 & 20 \\
Hypokinetic left ventricle & 4 & 4 \\
Wall motion not assessed & 12 & 13 \\
Loculated effusion & 1 & 1 \\
Electrocardiograph & & \\
Low voltage in limb leads & 62 & 67 \\
Nonspecific T-wave abnormalities & 50 & 54 \\
Atrial fibrillation/flutter & 13 & 14 \\
Atrial premature beats & 8 & 9 \\
ST-segment elevation & 7 & 8 \\
Electrical alternans & 4 & 4 \\
Left bundle branch block & 1 & 1 \\
Right bundle branch block & 1 & 1 \\
Normal & 3 & 3 \\
Not done & 2 & 2 \\
Chest radiography & & \\
Enlarged heart shadow & 75 & 81 \\
Pleural effusions & 64 & 69 \\
Parenchymal malignant disease & 38 & 41 \\
Congestive heart failure & 6 & 7 \\
\hline
\end{tabular}

radiography were enlargement of the cardiac silhouette, found in 75 patients $(81 \%)$, and pleural effusions, noted in 38 patients ( $41 \%$ ).

As outlined in Table IV, eight patients (8\%) did not receive tetracycline or doxycycline sclerosis for technical reasons. In five cases, the catheter became clotted before sclerosis could be attempted. In one case, the catheter could not be inserted; in another, the catheter was removed after complete drainage but before instillation of tetracycline because of discomfort at the catheter site. The eighth patient died after a cardiac arrest 1 hour after pericardiocentesis, before instillation of tetracycline. The remaining 85 patients received between one and eight instillations of tetracycline or doxycycline (median three) and a total dose of 500 to $7000 \mathrm{mg}$ (median dose $2000 \mathrm{mg}$ ). The median number of days required for sclerosis was 5 (range 1 to 17).

The complications of treatment are outlined in Table V. Two patients who had severe tamponade had cardiac arrests unrelated to sclerosis during pericardiocentesis. One patient was resuscitated and underwent sclerosis successfully, but the second could not be resuscitated. Retrosternal chest pain necessitating narcotic analgesia was seen in 17 patients $(20 \%)$. Seven patients had elevation of temperature to greater than $38.5^{\circ} \mathrm{C}$. In one patient, 
Table IV. Tetracycline or doxycycline instillations

\begin{tabular}{cc}
\hline & $\begin{array}{c}\text { No. of } \\
\text { patients }\end{array}$ \\
\hline No. of instillations (median 3) & 8 \\
0 & 21 \\
1 & 13 \\
2 & 21 \\
3 & 23 \\
4 & 4 \\
5 & 2 \\
$>5$ & \\
Dose of tetracycline (mg; median $1500 \mathrm{mg})$ & 8 \\
0 & 7 \\
500 & 1 \\
700 & 16 \\
1000 & 17 \\
1500 & 23 \\
2000 & 6 \\
3000 & 6 \\
3500 & 2 \\
4000 & \\
5000 & 4 \\
7000 & 1 \\
& 1
\end{tabular}

fever persisted after 24 hours and cultures of draining fluid yielded Staphylococcus aureus. Eight patients $(9 \%)$ had transient asymptomatic atrial arrhythmias (atrial fibrillation or flutter in five patients, paroxysmal atrial tachycardia in two). The catheter became occluded by clot after sclerosis in eight patients. These clots was successfully lysed by urokinase in four patients, by heparin in two patients, and by repeated saline solution flushes in one patient. No patient had pericardial constriction related to sclerosis therapy.

The results of tetracycline or doxycycline sclerosis are presented in Table VI. Of the 93 patients referred for sclerosis, eight did not undergo sclerosis because of the technical failures described previously. Of the remaining 85 patients, $68(79 \%)$ met the Smith criteria ${ }^{10}$ for control of MPE for longer than 30 days. Seven other patients had continuous control of the effusions but died of progressive malignant disease less than 30 days after sclerosis. Overall, $75(80 \%)$ of the original 93 patients (88\% of those who underwent sclerosis) achieved control of MPE, and their overall median survival was 98 days after sclerosis (range 1 to 1724 days). In these cases, all deaths resulted from progression of the underlying malignancy and were unrelated to MPE. Patients with breast cancer had a median survival of 131 days (range 6 to 1724 days), compared with 78
Table V. Complications of tetracycline or doxycycline sclerosis

\begin{tabular}{lrr}
\hline & No. & $\%$ \\
\hline Pain & 17 & 20 \\
Temperature $>38.5^{\circ} \mathrm{C}$ & 7 & 8 \\
Infection & 1 & 1 \\
Atrial fibrillation or flutter & 6 & 7 \\
Paroxysmal atrial tachycardia & 2 & 2 \\
Catheter plugged & 8 & 10 \\
Development of rub & 2 & 2 \\
Cardiac arrest before sclerosis & 2 & 2
\end{tabular}

days (range 3 to 828 days) for patients with lung cancer $(p<0.05)$.

Among the 18 patients who did not achieve control of their MPE, nine had no further treatment. Their median survival was 21 days (range $<1$ to 285 days). One patient had local radiation to the pericardium and survived 46 days; another, who had metastatic breast cancer, received chemotherapy and survived 455 days. Seven patients underwent surgical procedures, which included a total pericardiectomy for one patient (survival 5 days) and pericardial window formation for six. The median survival for the seven surgical patients was 21 days (range 5 to 90 days). The overall median survival for the 18 patients whose sclerosis failed was 21 days.

\section{Discussion}

The accumulation of fluid in the pericardial space is a potentially fatal complication of malignancy if not recognized early and treated aggressively. It has been reported that $36 \%$ of patients with metastases to the pericardium die as a direct result of pericardial involvement; in a further $49 \%$ of cases, pericardial involvement contributes significantly to death. ${ }^{2}$

The pathogenesis of MPE is thought to involve two major mechanisms. ${ }^{11}$ Increased fluid may be produced from the visceral pericardium as a result of direct spread of the tumor to the serosal surface and of fluid accumulation in the pericardial space. Alternatively, obstruction of lymphatic and venous outflow by tumor may cause increased hydrostatic pressure, which results in accumulation of fluid. Regardless of the mechanism that leads to the development of the MPE, the goals of treatment are to provide relief of symptoms and to prevent the immediate and late complications of this disorder, especially fatal cardiac tamponade.

In most cases, the diagnosis of MPE is made after the diagnosis of an underlying malignancy has been 
Table VI. Results of sclerosis in 93 patients with $M P E$

\begin{tabular}{lrr}
\hline & No. & $\%$ \\
\hline Technical failure & 8 & 8 \\
Sclerosis attempted & 85 & 92 \\
MPE controlled by sclerosis & 75 & 81 \\
Survived $>30$ days without recurrence & 68 & 73 \\
Survived <30 days without recurrence & 7 & 8 \\
Effusion uncontrolled by sclerosis & 11 & 13 \\
$\quad$ Primary failure of sclerosis & 5 & 5 \\
Rapid progression of malignancy & 4 & 4 \\
$\quad$ Recurrences & 3 & 3 \\
Salvage procedures & & \\
$\quad$ Pericardial window & 7 & 8 \\
Pericardiectomy & 1 & 1 \\
Pericardial radiation & 1 & 1 \\
Overall survival (days) & & \\
Median & 98 & \\
Range & $1-1724$ & \\
\hline
\end{tabular}

established. ${ }^{12}$ This was also the case in our study, in which only two patients had MPEs at the time of the initial cancer diagnosis. As a result, pericardial effusions frequently remain clinically unsuspected because the early symptoms may be nonspecific and attributed falsely to tumor progression. Furthermore, when MPE results in cardiac tamponade, the predominant symptoms are usually respiratory and may initially be attributed to worsening pulmonary or pleural metastases. ${ }^{2,13,14}$ As seen in our study, dyspnea, cough, and orthopnea were the most common presenting symptoms of MPE. The physical, electrocardiographic, and chest radiographic findings were generally nonspecific. The diagnosis was usually suspected when an enlarged cardiac shadow was seen on chest radiography; it was then confirmed in each case by echocardiography. All patients had a moderate to large pericardial effusion, and almost all had echocardiographic evidence of tamponade.

Although simple pericardiocentesis may be lifesaving, this procedure alone is seldom adequate as treatment because it is associated with a high rate of reaccumulation of fluid and recurrence of symptoms. ${ }^{15}$ For that reason, pericardiocentesis is usually followed by a definitive medical or surgical procedure designed to prevent further fluid accumulation and to provide an alternative drainage site to prevent cardiac tamponade.

Definitive management remains controversial. Surgical techniques for the management of MPE have evolved considerably during the past decade. Pericardiectomy is rarely performed today for MPE
Table VII. Comparison with published data

\begin{tabular}{lrccc}
\hline & This & & & \\
& series & Sclerosis* & Window $\dagger$ & VATS \\
\hline No. of patients & 93 & 64 & 28 & 49 \\
Breast and lung (\%) & 76 & $60-100$ & $61-100$ & $50-100$ \\
Local anesthetic (\%) & 100 & 100 & $56 \S$ & 0 \\
General anesthetic (\%) & 0 & 0 & $44 \S$ & 100 \\
30-day mortality (\%) & 7 & $0-10$ & $18-36$ & $0-38$ \\
Survival (days) & 98 & $104-230$ & $30-90$ & NA \\
Recurrence (\%) & 3 & $0-11$ & $0-29$ & 0 \\
Constriction (\%) & 0 & 0 & $0-10$ & 0 \\
\hline
\end{tabular}

VATS, Video-assisted thoracoscopic surgery; $N A$, not applicable.

${ }^{*}$ Data included from all patients reported in three studies of pericardial drainage and tetracycline sclerosis for initial management of MPE between 1984 and $1991 .{ }^{3,10}, 15$

$¥$ Data included from all patients reported in 11 studies of pericardial window formation for initial management of MPE between 1984 and $1995.5,6,17-25$

$\grave{\text { T} D a t a}$ included from all patients reported in seven studies of window formation by video-assisted thoracoscopic surgery. $8,9,26-30$

$\S$ Does not include data from one study in which patients with cancer were not separated from total number of patients when reporting percentage of procedures done with general anesthesia $(72 \%)$ and local anesthesia $(28 \%)^{25}$

because the operative risks range from $17 \%$ to $30 \% .^{16,17}$ Recent literature favors the creation of a pericardial window either by the subxiphoid route, by anterior thoracotomy, or by thoracoscopy. $8,9,17,18$ Indeed, thoracoscopy has led surgeons to a renewed interest in the surgical management of MPE, even though this technique requires general anesthesia and single-lung ventilation. ${ }^{9}$

We reviewed the results from all surgical series published in the last 15 years and extracted the outcomes reported for patients who underwent subxiphoid pericardial window creation with tube drainage ${ }^{5,6,17-25}$ or drainage by video-assisted thoracoscopic surgery. ${ }^{8,9,26-30}$ We compared the outcomes of these two techniques with results from published trials of tetracycline sclerosis ${ }^{3,10,15}$ and with the data from our series of 93 patients (Table VII). Direct comparison indicates that simple sclerosis provides similar survival with lower mortality and recurrence rates. The rates of pericardial constriction with the two procedures are comparable; indeed there are no cases reported after sclerosis, whereas our review indicates a $10 \%$ constriction rate in one study employing the pericardial window technique. The follow-up period for the thoracoscopic studies was shorter on average than that reported for the other techniques; long-term complications of recurrence and constriction associated with thoracoscopy may thus be underestimated.

Thoracoscopic pericardial window formation al- 
lows the surgeon to visualize the pleural space and the pericardium and provides the ability to perform biopsy procedures when necessary. Thoracoscopy may have an advantage in the treatment of undiagnosed malignancy because it provides excellent visualization of the thoracic cavity and because of the ability to perform diagnostic biopsy procedures. Most patients with MPE however, have a diagnosis of malignancy before the appearance of the effusion (96 of 98 in this series). In addition, rate of positive results of cytologic examination of pericardial fluid, reported to range from $57 \%$ to $100 \%$ among patients with malignancy, ${ }^{31-33}$ was $77 \%$ in our study. In studies that examined the correlation between fluid cytologic and pericardial biopsy results, the biopsy provided additional information in fewer than $20 \%$ of cases and failed to make the diagnosis in more than $50 \%$. In several studies that compared the results of cytologic examination and biopsy, the percentage of positive biopsy results among patients with positive cytologic results ranged from $33 \%$ to $55 \%$, and biopsy results were positive in only $0 \%$ to $21 \%$ of patients who had negative cytologic results. ${ }^{13,20-22}$

Our review suggests that the major differences between medical and surgical management are the operative risks associated with surgical procedures. Forty-four percent of the subxiphoid window creations and $100 \%$ of the video-assisted thoracoscopic procedures were performed with the patient under general anesthesia. Intraoperative complications reported in several series included hypotension necessitating inotropic support, ${ }^{21}$ pneumothorax, ${ }^{18}$ and death. $^{5,20}$ The development of atrial arrhythmias has been reported in both surgical ${ }^{5}$ and medical series $^{3,15}$ and was seen in $7 \%$ of the cases in our series. These arrhythmias were always self-limited and never life-threatening. Pain and fever, which have been cited as unacceptable accompaniments of percutaneous drainage and sclerosis, ${ }^{19}$ were seen in $20 \%$ and $7 \%$ of patients, respectively, in our series. The fever usually lasted less than 24 hours, and an organism was cultured from the pericardial fluid in only one case in which the catheter remained in place for longer than 7 days. Pain was temporally related to the injection of the sclerosing agent; despite premedication with lidocaine of all patients, it was necessary to administer narcotic analgesics to 17 patients $(20 \%)$. Pain and fever are also common occurrences during the early postoperative period after almost all surgical procedures, however, and we believe that low rates of these complications are reported in the surgical series because they are not considered to be complications worthy of note.

Because the parenteral form of tetracycline is no longer commercially available, other sclerosing agents are currently under investigation. Both doxycycline and bleomycin have been used successfully ${ }^{34,35}$ in small series, and we are currently undertaking a prospective comparison of these two agents.

In summary, percutaneous pericardiocentesis and tetracycline or doxycycline sclerosis is a safe and effective safe treatment for MPE. In patients with known malignancy or with positive results of cytologic examination of pericardial fluid, sclerosis appears to be superior to subxiphoid pericardial window formation and video-assisted thoracoscopy. The latter technique should be reserved for those cases that remain undiagnosed after pericardiocentesis or for the small percentage of cases in which it is not possible to control the effusion by percutaneous drainage and sclerosis.

\section{REFERENCES}

1. Theologides H. Neoplastic cardiac tamponade. Semin Oncol 1978;5:181-90.

2. Thurber D, Edwards J, Anchor R. Secondary malignant tumors of the pericardium. Circulation 1962;26:228-41.

3. Davis S, Rambotti P, Griznani F. Intrapericardial tetracycline sclerosis in the treatment of malignant pericardial effusion: an analysis of thirty-three cases. J Clin Oncol 1984;2:631-6.

4. Shepherd F, Morgan C, Evans W, Ginsberg J, Watt D, Murphy K. Medical management of malignant pericardial effusion by tetracycline sclerosis. Am J Cardiol 1987;60: 1161-6.

5. Chan A, Rischin D, Clarke C, Woodruff R. Subxiphoid partial pericardiectomy with or without sclerosant instillation in the treatment of symptomatic pericardial effusions in patients with malignancy. Cancer 1991;68:1021-5.

6. Okamoto H, Shinkai $T$, Yamakido M, Saijo N. Cardiac tamponade caused by primary lung cancer and the management of pericardial effusion. Cancer 1993;71:93-8.

7. McIntosh I, Jones E. Treatment of malignant pericardial effusion [abstract]. Chest 1994;27(Suppl 1):1629.

8. Hazelrigg SR, Mack MJ, Landreneau RJ, Acuff TE, Seifert PE, Auer JE. Thoracoscopic pericardiectomy for effusive pericardial disease. Ann Thorac Surg 1993;56:792-5.

9. Mack MJ, Landreneau RJ, Hazelrigg SR, Acuff TE. Video thoracoscopic management of benign and malignant pericardial effusions. Chest 1993;103:390S-3S.

10. Smith F, Lane M, Hudgins P. Conservative management of malignant pericardial effusion. Cancer 1974;33:47-57.

11. Missri J, Schechter D. When pericardial effusion complicates cancer. Hosp Practice [Off] 1988;23:277-81, 284-6.

12. Fraser $R$, Viloria J, Wang N. Cardiac tamponade as a presentation of extracardiac malignancy. Cancer 1980;45: $1697-704$

13. Reitknecht F, Regal A, Antkowiak J, Takita H. Management 
of cardiac tamponade in patients with malignancy. J Surg Oncol 1985;30:19-22.

14. Rinkevich D, Borovik R, Bendett M, Markiewicz W. Malignant pericardial tamponade. Med Pediatr Oncol 1990;18:28791.

15. Celermajer D, Boyer M, Bailey B, Tattersall M. Pericardiocentesis for symptomatic malignant pericardial effusion: a study of 36 patients. Med J Aust 1991;154:19-22.

16. Palatianos $G$, Thurer R, Kaiser L. Comparison of effectiveness and safety of operations on the pericardium. Chest 1985:88:30-3.

17. Piehler J, Pluth J, Schaff H, Danielson G, Orszulak T, Puga F. Surgical management of effusive pericardial disease. Influence of extent of pericardial resection on clinical course. $\mathbf{J}$ Thorac Cardiovasc Surg 1985;90:506-16.

18. Park J, Rentschler R, Wilbur D. Surgical management of pericardial effusion in patients with malignancies: comparison of subxiphoid window versus pericardiectomy. Cancer 1991;67:76-80

19. Hankins J, Satterfield J, Aisner J, Wiernik P, McLaughlin J. Pericardial window for malignant pericardial effusion. Ann Thorac Surg 1980;30:465-71.

20. Mills S, Julian S, Holiday R, Vinten J, Case L, Hudspeth A, et al. Subxiphoid pericardial window for pericardial effusive disease. J Cardiovasc Surg 1989;30:768-73.

21. Campbell P, Van Trigt P, Wall T, Kenney R, O'Connor C, Sheikh K, et al. Subxiphoid pericardiotomy in the diagnosis and management of large pericardial effusions associated with malignancy. Chest 1992;101:938-43.

22. Osuch J, Khandekar J, Fry W. Emergency subxiphoid pericardial decompression for malignant pericardial effusion. Am Surg 1985;51:298-300.

23. Sugimoto J, Little A, Ferguson M, Borrow K, Vallera D, Staszak V, et al. Pericardial window: mechanisms of efficacy. Ann Thorac Surg 1990;50:442-5.

24. Bitran J, Evans R, Brown C. The management of cardiac tamponade in patients with breast cancer. J Surg Oncol 1984;27:42-4.
25. Moores DW, Allen KB, Faber LP, Dziuban SW, Gillman DJ, Warren WH, Ilves R, Lininger L. Subxiphoid pericardial drainage for pericardial tamponade. $\mathbf{J}$ Thorac Cardiovasc Surg 1995;109:546-52.

26. Mann GB, Nguyen H, Corbet J. Laparoscopic creation of pericardial window. Austr N Z J Surg 1994;64:853-5.

27. Liu HP, Chang CH, Lin PJ, Hsieh HC, Chang JP, Hsieh MJ. Thoracoscopic management of effusive pericardial disease: indications and treatment. Ann Thorac Surg 1994;58: $1695-7$.

28. van der Schelling GP, Michielsen D, Hendrickx L, Hubens A. Thoracoscopic pericardial fenestration, a first experience of a new approach. Acta Chir Belg 1994;94:272-3.

29. Shapira OM, Aldea GS, Fonger JD, Shemin RJ. Videoassisted thoracic surgical techniques in the diagnosis and management of pericardial effusion in patients with advanced lung cancer. Chest 1993;104:1262-3.

30. Canto A, Guijarro R, Arnau A, Fernandez-Centeno A, Ciscar MA, Galbis J, et al. Thoracoscopic pericardial fenestration: diagnostic and therapeutic aspects. Thorax 1993;48: 1178-80.

31. Posner M, Cohen G, Skarin A. Pericardial disease in patients with cancer: the differentiation of malignant from idiopathic and radiation-induced pericarditis. Am J Med 1981;71:40713.

32. Wiener $\mathrm{H}$, Kristensen $\mathrm{O}$, Hanbek A, Kristensen B, Baandrup $\mathrm{U}$. The diagnostic value of pericardial cytology: an analysis of 95 cases. Acta Cytol 1991;35:149-53.

33. Edoute Y, Malberger E, Kuten A, Ben-Haim S, Moscovitz M. Cytologic analysis of pericardial effusion complicating malignancy. Am J Cardiol 1992;69:568-71.

34. Vaitkus PT, Hermann HC, LeWinter MM. Treatment of malignant pericardial effusions. JAMA 1994;272:59-64.

35. Yano $\mathrm{T}$, Yokoyama $\mathrm{H}$, Inoue $\mathrm{T}$, Takanashi $\mathrm{N}$, Asoh $\mathrm{H}$, Ichinose $\mathrm{Y}$. A simple technique to manage pericardial effusion with a local instillation of bleomycin in non-small cell carcinoma of the lung. Oncology 1994;51:507-9.

\section{Availability of Journal back issues}

As a service to our subscribers, copies of back issues of The Journal of Thoracic and Cardiovascular Surgery for the preceding 5 years are maintained and are available for purchase from Mosby at a cost of $\$ 13.50$ per issue until inventory is depleted. The following quantity discounts are available: $25 \%$ off on quantities of 12 to 23 , and one third off on quantities of 24 or more. Please write to Mosby-Year Book, Inc., Subscription Services, 11830 Westline Industrial Drive, St. Louis MO 63146-3318, or call 800-453-4351 or 314-453-4351 for information on availability of particular issues. If unavailable from the publisher, photocopies of complete issues may be purchased from UMI, 300 N. Zeeb Rd., Ann Arbor, MI 48106, 313-761-4700. 